\title{
REMINERALIZATION POTENTIAL OF GINGER AND ROSEMARY HERBALS VERSUS SODIUM FLUORIDE IN TREATMENT OF WHITE SPOT LESIONS: A RANDOMIZED CLINICAL TRIAL
}

\author{
Sara M. Hassan*, Amir Hafez** and Maha A. Elbaz**
}

\begin{abstract}
Aim: The current study was conducted to evaluate the effectiveness of ginger and rosemary herbals versus sodium fluoride in remineralization of incipient enamel caries.

Methodology: white spot lesions in anterior teeth of post orthodontic debanding patients received randomly two types of remineralizing agents, either ginger and rosemary gel or sodium fluoride varnish (bifluorid 10). White spot lesions were evaluated at base line one month and after 3,6 months follow up periods by two blinded assessors to assess white spot lesion progression by Kavo DIAGNOdent.
\end{abstract}

Results: Mann-Whitney test showed that there at baseline, sodium fluoride varnish group yielded similar readings as ginger and rosemary gel group $(\mathrm{P}=0.060)$. While at 1 month, ginger and rosemary gel group showed significantly higher readings compared to sodium fluoride varnish group $(\mathrm{P}<0.001)$. However, there were no statistically significant differences in readings of both groups at 3 months $(\mathrm{P}=0.302)$ and 6 months $(\mathrm{P}=0.070)$.

Conclusion: Fluoride varnish and ginger and rosemary gel can decrease post-orthodontic white spot lesions. The herbals ginger and rosemary may be preferable for prevention purposes on initial remineralization of enamel lesions as more natural products are preferred today.

KEYWORDS: White spot lesions, ginger and rosemary in initial caries, sodium fluoride varnish, DIAGNOdent.

\section{INTRODUCTION}

Dental caries is one of the most common oral diseases found worldwide. It is a process of demineralization of tooth structure by acids formed from bacterial fermentation of dietary carbohydrates Anitha et al. (2016) ${ }^{1}$.

White spot lesions (WSL) is defined as a 'subsurface enamel porosity from carious

* MSc Degree Candidate, Conservative Dentistry Department, Faculty of Dentistry, Cairo University

** Associate Professor, Conservative Dentistry Department, Faculty of Dentistry, Cairo University 
demineralization' that appears like 'a milky white opacity when located on smooth surfaces'. The WSL is the first visible evidence of caries in enamel, characterized by a demineralized lesion underneath an intact surface. WSL develop as a result of an imbalance between demineralization and remineralization of the enamel. The lesion is caused by the accumulation of plaque and bacteria, along with insufficient oral hygiene. High oral plaque accumulation can increase the risk of WSL development Zoha and Joseph (2015) ${ }^{15}$.

Demineralization occurs around fixed orthodontic brackets as oral hygiene becomes more difficult. This decalcification is manifested as white spot lesions. If these lesions are not treated, it can progress to produce cavitations. In addition, white spot lesions affect the esthetic appearance of the patient Bishara and Ostby (2008) ${ }^{4}$.

The preliminary choice for the elimination of WSLs is remineralization which, apart from strict oral hygiene measures, involves repeated applications of home applied products or professionally applied products which might take a long time. In contrast to cavitated caries lesions, WSL can be reversed by re-establishing a balance between demineralization and remineralization. Several remineralizing agents have demonstrated a positive effect on WSL including sodium fluoride, CPP-ACP, and natural products like grape seed extract, ginger, honey, and rosemary Bilgin et al. (2016) ${ }^{3}$.

Sodium fluoride varnish is considered the gold standard for remineralization of white spot lesions however, has many drawbacks one of them is that high concentration of fluoride application to WSLs is usually preferred; however, high fluoride concentration leads to hypermineralization of surface layer of WSLs. Therefore, the penetration of calcium and phosphorus into the body of the lesion becomes blocked and may have some undesirable esthetics. Guclu et al. (2016) ${ }^{7}$.
Natural products have fewer side effects, better patient tolerance, relatively less expensive, acceptance due to its long history of use and being renewable in nature. Moreover, ginger is as very potent antioxidant, anti-inflammatory, antibacterial and antifungal, immune boosting agent, and to sedate dental pain. Also rosemary has an antibacterial, antifungal, antioxidant, and anti-inflammatory properties. In addition, it is used in relieving toothache. Anushri et al. (2015) ${ }^{2}$.

The null hypothesis tested in this study, that there is no difference between sodium fluoride varnish and the natural herbs ginger and rosemary gel in treatment of early white spot lesions.

\section{MATERIALS AND METHODS}

The materials used in this study were:

BiFluorid 10(control). It is a varnish, which has $5 \%$ sodium fluoride (equal to 22.600-ppm fluoride) and $5 \%$ calcium fluoride (Voco, Germany).

Ginger and rosemary gel (Intervention): Ginger and rosemary powder preparation: The ginger and rosemary used in this research are purchased from the local market. The fresh ginger and rosemary (250 g each) will be washed with tape water, chopped into 1-2 $\mathrm{mm}$ size pieces and macerated in Ethyl alcohol for 12 hours at $40^{\circ} \mathrm{C}$. The combined Ethanolic extract will be evaporated to dryness under reduced pressure at a temperature not exceeding $40^{\circ} \mathrm{C}$ using rotatory evaporator (Heidolph, Germany). Sejali and Anuar (2011) ${ }^{12}$.

Preparation of ginger and rosemary extract hydrogel formulations

Solution of a $0.5 \%$ concentration of ginger and rosemary extract will be prepared through dissolving $50 \mathrm{mg}$ of the dried powdered extract in aqueous ethanol $(20 \% \mathrm{v} / \mathrm{v})$ and completing the volume to $10 \mathrm{ml}$ to yield a solution of concentration $0.5 \%$. Namir and Wesal (2011). The ginger extract hydrogels will be prepared through dissolving 
Sodium carboxymethyl cellulose, the gelling agent, in the previously prepared solutions $(5-10 \% \mathrm{w} / \mathrm{v})$ under continuous stirring at $1000 \mathrm{rpm}$ using a magnetic stirrer. The formed hydrogels will be kept at $8^{\circ} \mathrm{C}$ till further use ${ }^{11}$.

The trail protocol was designed following the SPIRIT 2013 statement and approved from Evidence Based Dentistry Committee, Faculty of Dentistry Cairo University .The protocol and the template of informed consent form were reviewed and approved by the Ethics Committee of scientific research, Faculty of Dentistry - Cairo University in June 2019 (Approval No.19617). The trail was registered in Protocol registration and results system (ClinicalTrails.gov PRS) with an identification number NCT03990935.

Regarding the design, it is a single Centered, triple blinded (participant, assessor and the data analyzer were blinded); two parallel armed randomized control clinical trial. The study was carried out on 58 adult Participants (29 in each group) attending to the operative clinic in The Faculty of Dentistry, Cairo University, Egypt. Each participant has chosen a sequentially numbered opaque sealed envelope.

\section{Eligibility criteria:}

Inclusion criteria of participants: Du et al. (2011) ${ }^{6}$.

- No gender restriction (Males or Females).

- Patient who has just completed a comprehensive orthodontic treatment.

- Presence of at least one WSL, DIAGNOdent score (0-20).

- Age range between 13 and 35years.

Exclusion criteria of participants: Du et al. $(2011)^{6}$

- Allergic to the ingredients used in the study.

- Patients with a compromised medical history.

- Severe or active periodontal disease.

- Patients with developmental hypo-calcified lesions.

\section{Clinical procedures:}

Each participant received both types of the remineralizing agents according to the randomization done and white spot lesions were assessed before (baseline), one month later, 3 months later and six months later.

\section{Application of Ginger and Rosemary gel:}

After opening the sealed envelope and knowing the code of participants, while the cheek retractor was in place, teeth surfaces were cleaned with a polishing brush with paste, rinsed, and dried thoroughly. Then, application of a thin ribbon of this gel to the teeth using a brush. The medication will be left for 10 minutes. The participant will be asked to spit out the medication after use and not to swallow it. Also the participant will be asked not to rinse his/her mouth, eat, or drink for at least 30 minutes after use to get the best results.

\section{Application of sodium fluoride varnish:}

BiFluorid 10 (VOCO) was the used sodium fluoride varnish. The single dose form was used, for optimal standardization of the amount of fluoride varnish used. While, the cheek retractor was in place, teeth surfaces were cleaned with a polishing brush with paste, rinsed, and dried thoroughly. The foil in the single dose unit was pierced using a Micro-Tim brush, the opening was enlarged, and in a circular movement, the brush was being coated with the fluoride varnish. Thin coat was applied on the cleaned teeth in the side allocated for sodium fluoride treatment. The varnish left undisturbed from 10-20 seconds then air-dried.

\section{Postoperative evaluation:}

The change in the lesion fluorescence will be evaluated using diode laser fluorescence device (DIAGNOdent)

\section{Laser fluorescence assessment:}

Based on principle of fluorescence, it uses a diode laser light source and a fiber optic cable that transmits light to a hand held probe. Light is 
absorbed induces infrared fluorescence by organic and inorganic materials. Emitted fluorescence is collected at probe tip, processed and presented on display as an integer between 0 and 99 .

Based on the previous study by Yazıcıoğlu and Ulukapi (2014), laser fluorescence examination results had been scored using the manufacturer's scoring system ${ }^{14}$.

Score 1: Laser fluorescence score 0-4, no caries, or white opaque lesions

Score 2: Laser fluorescence score 5-10, enamel caries limited to the outer half of the enamel thickness

Score 3: Laser fluorescence score 11-20, enamel caries limited to the inner half of the enamel thickness without obvious spread in the dentin.

Score 4: Laser fluorescence score $\geq 21$, caries spread in the dentin.

\section{Statistical analysis:}

Statistical analysis was performed using IBM SPSS Statistics Version 2.0 for Windows. Data was presented as mean, standard deviation (SD), frequencies (n) and percentages. KolmogorovSmirnov and Shapiro-Wilk tests were used to assess data normality of continuous data. The significance level was set at $\mathrm{P} \leq 0.05$. Mann-Whitney test was performed for intergroup comparisons and KruskalWallis test was used for multiple comparisons. Chi-square test was used to compare frequency distribution of categorical data.

\section{RESULTS}

Mann-Whitney test (table 1and figure 1) showed that there at baseline, sodium fluoride varnish group yielded similar readings as ginger and rosemary gel group $(\mathrm{P}=0.060)$. While at 1 month, ginger and rosemary gel group showed significantly higher readings compared to sodium fluoride varnish group $(\mathrm{P}<0.001)$. However, there were no statistically significant differences in readings of both groups at 3 months $(\mathrm{P}=0.302)$ and 6 months $(\mathrm{P}=0.070)$.

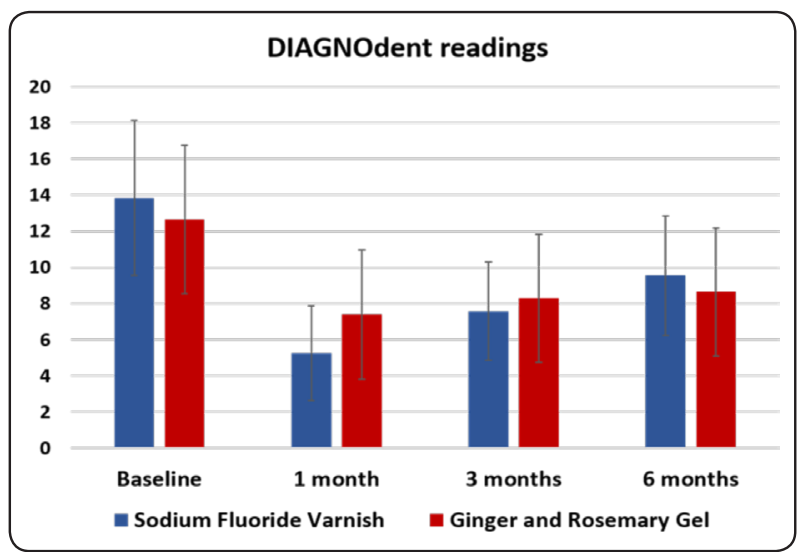

Fig. (1): Bar chart showing DIAGNOdent readings between remineralizing agents at each evaluation time.

Chi-square (table 2 and figure 2) showed that there was a statistically significant difference in score distribution between sodium fluoride varnish and ginger and rosemary gel groups 1 month $(\mathrm{P}<0.001)$ and 3 months $(\mathrm{P}=0.041)$. While there was no statistically significant difference in score distribution between both groups at baseline $(\mathrm{P}=0.060)$, and at 6 months $(\mathrm{P}=0.083)$.

TABLE (1): Mean \pm SD and P-value for the comparison of DIAGNOdent readings between remineralizing agents at each evaluation time.

\begin{tabular}{|c|c|c|c|c|}
\hline & Baseline & 1 month & 3 months & 6 months \\
\hline Sodium Fluoride Varnish & $13.34 \pm 4.29$ & $5.27 \pm 2.62$ & $7.58 \pm 2.72$ & $9.54 \pm 3.31$ \\
\hline Ginger and Rosemary Gel & $12.65 \pm 4.11$ & $7.39 \pm 3.58$ & $8.29 \pm 3.53$ & $8.65 \pm 3.53$ \\
\hline P-value & $0.060 \mathrm{NS}$ & $<0.001^{*}$ & $0.302 \mathrm{NS}$ & $0.070 \mathrm{NS}$ \\
\hline
\end{tabular}


TABLE (2): Distribution of laser fluorescence score system within each remineralizing agent at different evaluation times.

\begin{tabular}{|c|c|c|c|c|c|}
\hline & & \multicolumn{3}{|c|}{ Scoring system (n/\%) } & \multirow{2}{*}{ P-value } \\
\hline & & Score 1 & Score 2 & Score 3 & \\
\hline \multirow{2}{*}{ Baseline } & Sodium Fluoride Varnish & $0(0 \%)$ & $30(27.2 \%)$ & $80(72.7 \%)$ & \multirow{2}{*}{$0.060 \mathrm{NS}$} \\
\hline & Ginger and Rosemary Gel & $0(0 \%)$ & $41(41.4 \%)$ & $58(58.5 \%)$ & \\
\hline \multirow{2}{*}{1 month } & Sodium Fluoride Varnish & $47(46.0 \%)$ & $53(51.9 \%)$ & $2(1.9 \%)$ & \multirow{2}{*}{$<0.001^{*}$} \\
\hline & Ginger and Rosemary Gel & $24(25.8 \%)$ & $47(50.5 \%)$ & $22(23.65 \%)$ & \\
\hline \multirow{2}{*}{3 months } & Sodium Fluoride Varnish & $17(16.6 \%)$ & $70(68.6 \%)$ & $15(14.7 \%)$ & \multirow{2}{*}{$0.041 *$} \\
\hline & Ginger and Rosemary Gel & $14(15.0 \%)$ & $52(55.9 \%)$ & $27(29.0 \%)$ & \\
\hline \multirow{2}{*}{6 months } & Sodium Fluoride Varnish & $3(2.9 \%)$ & $60(58.8 \%)$ & $39(38.2 \%)$ & \multirow{2}{*}{$0.083 \mathrm{NS}$} \\
\hline & Ginger and Rosemary Gel & $10(10.7 \%)$ & $52(55.9 \%)$ & $31(33.3 \%)$ & \\
\hline
\end{tabular}

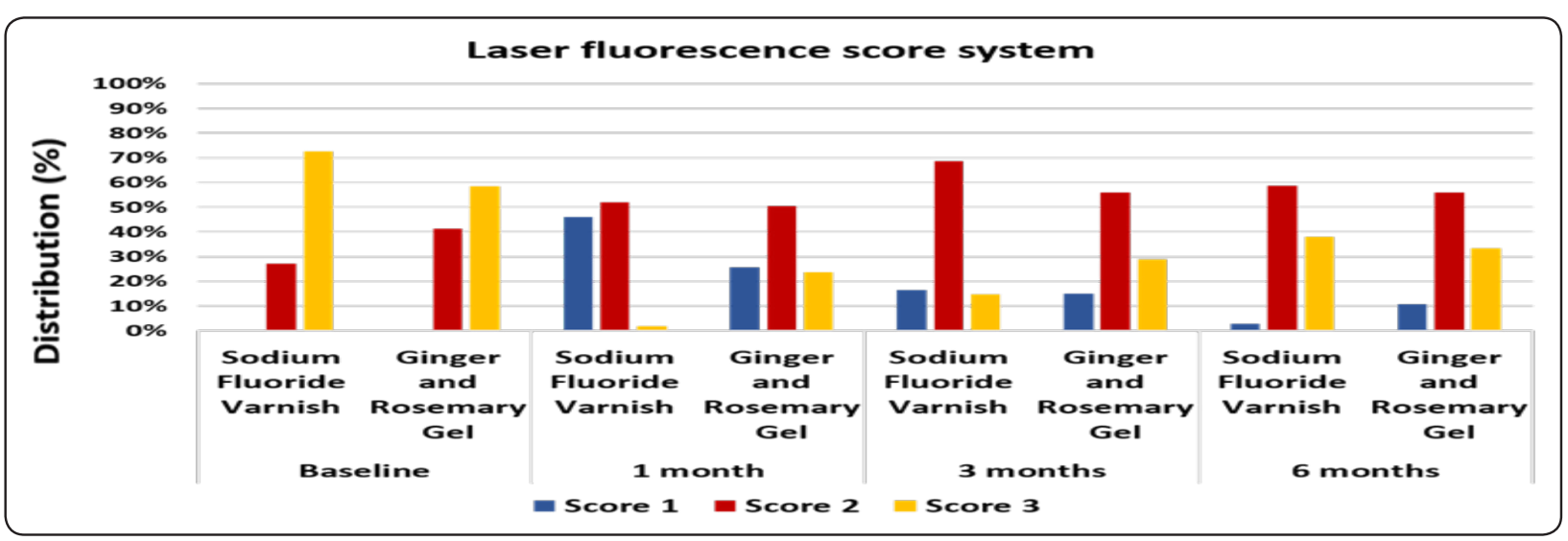

Fig. (2): Distribution (\%) of laser fluorescence score system within each remineralizing agent at different evaluation times.

\section{DISCUSSION}

Du et al. (2011) showed that fixed orthodontic appliances induced a rapid increase in the volume of dental plaque. The composition of the bacterial flora of plaques shifts rapidly after introducing orthodontic appliances ${ }^{6}$. It was observed that there was a significant elevation in plaque and salivary levels of acidogenic bacteria, such as Streptococcus Mutans and lactobacilli in orthodontic patients. The low $\mathrm{pH}$ of plaques adjacent to the orthodontic appliances and areas without optimal oral hygiene favors caries development; thus, decalcification of enamel and even carious cavity occurs. Moreover, because of low concentrations of calcium, phosphate, and fluoride in the saliva, natural remineralization after orthodontic treatment is difficult to achieve. Thus, intervention is needed to promote reversal of the caries process in the enamel lesions.

The complete elimination of WSLs is unlikely, and some WSLs last for up to 5-12 years. Therefore, it becomes necessary to apply remineralizing agents to repair the deeper parts of WSLs for better esthetics. The commonly used agents for the treatment of WSLs are topical fluorides. According 
to Bilgin et al. (2016), WSL can be reversed by reestablishing a balance between demineralization and remineralization. Several remineralizing agents have demonstrated a positive effect on WSL including sodium fluoride, CPP-ACP, and natural products like grape seed extract, ginger, honey, and rosemary ${ }^{3}$

The aim of this study was to evaluate the effectiveness of ginger and rosemary herbals versus sodium fluoride varnish (BiFluorid 10) in remineralization of incipient enamel caries. The sodium fluoride varnish considered as control agent because it is the most popular used remineralizing agent in the treatment of white spot lesions. The fluoride has many roles in inhibiting the demineralization, enhancing the remineralization and having a strong bactericidal effect. Active free ions of Fluoride when it becomes available in the oral environmental fluids, formation of fluorapatite and calcium fluoride take place. Deposition of fluorapatite crystals dictates the presence of fluoride, calcium and phosphate ions in an adequate amount. While for the formation of calcium fluoride, presence of only calcium and fluoride ions is needed. Furthermore, the concentration of fluoride in the oral fluid and its $\mathrm{pH}$ are other deciding factors for formation of fluorapatite crystals. When it becomes lower than $50-\mathrm{ppm}$ and $\mathrm{pH}$ is more than 4.5 , the circumstance becomes favorable to Fluorapatite formation. On the other hand, when the fluoride concentration is more than $100-\mathrm{ppm}$ and $\mathrm{pH}$ is less than 4.5 , calcium fluoride is formed as mentioned by Khoroushi and Kachuie (2017). It is also of great importance to know that, the solubility of fluorapatite in the oral fluids is less than calcium fluoride. Solubility of Calcium fluoride is beneficial in reducing the effect of acid attack during low $\mathrm{pH}$ as it release free ionic fluoride ions, which helps in the remineralization 9 .

On the other hand, ginger rhizome (Zingiber officinale Roscoe, Zingiberacae) and rosemary (Rosmarinus officinalis L., Lamiaceae) have been used as food spices and medicinal plants for centuries. Moreover, they are natural materials, showing no toxicity, and are considered 'generally recognized as safe' (GRAS) by the US Food and Drug Administration (FDA). Their antifungal and antimicrobial effects on oral cavity pathogens have been reported in many studies. The remineralization obtained was probably due to the antimicrobial properties and the high fluoride and calcium content of ginger. In addition, rosemary has inhibitory effects on S. mutans.it was found that the rosemarycontaining treatment mixture was effective in enhancing the remineralization process of enamel as discussed by Bilgin et al. in (2016) ${ }^{3}$.

The reason behind using $(0.5 \%)$ of ginger concentration is that there is a maximum amount of free calcium ions that can be deposited in the tooth surface, but above this concentration there may be a decrease in this amount of free calcium due to the increase in the concentrations of elements other than calcium as ginger is also rich in iron, magnesium, phosphorus, potassium. So the increase in the concentrations of the extract may increase the concentrations of these elements as well, in which they may substitute calcium ions from hydroxyapatite crystals in enamel surface as mentioned by Namir and wesal (2011) ${ }^{11}$.

The results of the current study revealed the sodium fluoride varnish is better than ginger and rosemary gel at $\mathrm{T}_{1}$ (after one month) with no significant difference in $\mathrm{T}_{2}$ (3 months) and $\mathrm{T}_{3}(6$ months later). This could be explained by Khoroushi and Kachuie (2017), as it was concluded that fluoride varnishes release fluoride at a slow rate for up to 6 months, with the greatest release observed during the first 3 weeks, followed by a more gradual delivery. Therefore, it is recommended a biannual application of single-dose fluoride varnish to gain optimum results. ${ }^{9}$.

Baseline readings displayed the significantly highest readings followed by 6 months, 3 months, then 1 month which showed the significantly least readings in both groups. This result was with 
agreement with Virupaxi et al. in (2016) who determined the longevity of fluoride release from three different fluoride varnishes over a period of time through salivary fluoride estimation ${ }^{13}$. It was found that the fluoride varnishes released fluoride for an extended period of time, with the greatest occurring in the first three weeks and then tapers. In addition, the Sodium fluoride varnish did not have a long lasting effect. It is removed gradually from the affected tooth surface by tooth brushing action or consumption of acidic drinks. This could be related to the solubility of the calcium fluoride that is highly soluble in relation to the fluorapatite crystals .This explains why the readings of the DIAGNOdent were getting higher in $\mathrm{T} 3$ and $\mathrm{T} 4$.

This was also an agreement with Huang et al. (2013), who warn against the use of high concentrations of fluoride because they believe this will cause remineralization mainly in the superficial part of the WSLs. The previously mentioned superficial layer could be able to prevent both calcium and phosphate from penetrating to the deeper layers of the enamel, thus inhibiting deeper remineralization and limiting the cosmetic improvement of the WSLs. Thus, the most ideal concentrations and delivery vehicles for fluoride remain controversial ${ }^{8}$.

However, Du et al. (2011) disagrees with the results of the current study. He discussed that the application of fluoride varnish associated with good oral hygiene and use of fluoridated toothpaste were sufficient to promote remineralization. It has been found that fluoride increases the initial rate of remineralization and recommended to use high doses of fluoride during and after orthodontic treatment for arresting areas of decalcification and preventing WSLs from progressing to carious lesions. This should cause an increase in remineralization of the outer enamel and a decrease in demineralization of the inner enamel, resulting in a significant mineral gain ${ }^{6}$.
Laser fluorescence (LF) is a method introduced for early diagnosis of the dental caries. It is useful for early detection of hidden caries in non-cavitated teeth through a non-invasive method. It emits infrared light $(655 \mathrm{~nm})$, that can be absorbed by organic and inorganic tooth materials, and the process of remitted fluorescence shows various scales between 0 and 99. DIAGNOdent had some disadvantages, such as the fact that readings could also be affected by stains, calculus, and plaque and were based on bacterial metabolites as mentioned by Khushboo et al. (2013) ${ }^{10}$.

By the end of the discussion our null hypothesis was accepted that there is no significant difference between the reminerlization potential of ginger and rosemary gel and fluoride varnish (bifluorid 10) on caries white spot lesions. According to the data obtained from this study it is possible to interpret that the ginger and rosemary gel can inhibit demineralization and/or promote remineralization of WSLs in the enamel and may serve as a promising alternative for the treatment of white spot lesion.

\section{CONCLUSIONS}

Within the limitations of this study the following conclusions could be drawn:

1. Fluoride varnish and ginger and rosemary gel can decrease post orthodontic white spot lesions.

2. Ginger and rosemary may be preferable for prevention purposes on initial remineralization of enamel lesions, as more natural products are preferred today.

\section{RECOMMENDATIONS}

1. Further clinical trials with multiple applications of the ginger and rosemary gel.

2. Maintaining good oral hygiene is the most important prophylactic measure in fixed orthodontic patients to prevent WSLs. 


\section{REFERENCES}

1. Anitha, L., and Sunita Raju, k. (2016). A Review on Antimicrobial Activity of Vegetables, Herbs and Spices Against Cariogenic Bacteria. Research Journal of Biology. 4(4) $12-20$.

2. Anushri,M., Yashoda, R., and Manjunath,P.(2015)."Herbs: A Good Alternatives to Current Treatments for Oral Health Problems". International Journal of Advanced Health Sciences. 12(1), 28-33.

3. Bilgin, G., Yanıkoğlu, F., and Tagtekin, D. (2016) Remineralization Potential of Herbal Mixtures: An In Situ Study. Indian journal of research.5 (2), 264-268.

4. Bishara, S. E., and Ostby, A. W. (2008).White Spot Lesions: Formation, Prevention, and Treatment. Seminars in Orthodontics.14 (3), 174-182.

5. Celiberti, P., Leamari, V.M., Imparato, J.C., Braga, M.M., Mendes, F.M. (2010) In vitro ability of a laser fluorescence device in quantifying approximal caries lesions in primary molars. Journal of Dentistry.38(8):666-70.

6. Du, M., Cheng, N., Tai, B., Jiang, H., Li, J., and Bian, Z. (2011). "Randomized controlled trial on fluoride varnish application for treatment of white spot lesion after fixed orthodontic treatment”. Clinical Oral Investigations.16 (2), 463-468.

7. Guclu, Z., Alacam, A., and Coleman, N. (2016). “A 12 week assessment of the treatment of white spot lesions with CPP-ACP paste and/or fluoride varnish". BioMed research international. 2(16), 1-9.

8. Huang, G. J., Roloff-Chiang, B., Mills, B. E., Shalchi, S., Spiekerman, C., Korpak, A. M., and Matunas, J. C. (2013).
Effectiveness of MI Paste Plus and PreviDent fluoride varnish for treatment of white spot lesions: a randomized controlled trial. American Journal of Orthodontics and Dentofacial Orthopedics, 143(1), 31-41.

9. Khoroushi, M., and Kachuie, M. (2017). Prevention and treatment of white spot lesions in orthodontic patients. Contemporary clinical dentistry, 8(1), 11-16.

10. Khushboo, S., Ashish, S., Basavaraj, P., and Shilpi, S. (2013). "Diagnodent- in detection of dental decay - A systematic review". Journal of pearldent. 4(4), 7-17.

11. Namir, S., and Wesal A. (2011)."An evaluation of ultrasonic water extract of ginger on microhardness and microscopic features of enamel caries like lesions, compared to fluoridated agent (An in vitro study)". Journal of Baghdad College Dentistry. 23(4).148-152.

12. Sejali, S. N. F., \& Anuar, M. S. (2011). Effect of Drying Methods on Phenolic Contents of Neem (Azadirachta indica) Leaf Powder. Journal of Herbs, Spices \& Medicinal Plants, 17(2), 119-131.

13. Virupaxi, SG., Roshan, NM., Poornima, P., Nagaveni, NB., Neena, IE., and Bharath, KP.(2016). "Comparative Evaluation of Longevity of Fluoride Release From three Different Fluoride Varnishes - An In vitro Study”. Journal of clinical and diagnostic research. 10(8): ZC33-ZC36.

14. Yazıcıoğlu, O., \& Ulukapı, H. (2013). The investigation of non-invasive techniques for treating early approximal carious lesions: an in vivo study. International Dental Journal, 64(1), 1-11.

15. Zoha, A., and Joseph, J. (2015).”Minimally Invasive Treatment of White Spot Lesions.A Systematic Review". 14(3)197-198. 